\title{
Recursive Priority Inheritance Protocol for Solving Priority Inversion Problems in Real-Time Operating Systems ${ }^{\star}$
}

\author{
Kwangsun Ko, Seong-Goo Kang, Gyehyeon Gyeong, and Young Ik Eom \\ School of Information and Communication Eng., Sungkyunkwan University, \\ 300 Cheoncheon-dong, Jangan-gu, Suwon, Gyeonggi-do 440-746, Korea \\ $\{$ rilla91,lived, gyehyeon, yieom\}@ece.skku.ac.kr
}

\begin{abstract}
In this paper, a protocol, called recursive priority inheritance (RPI) protocol, is proposed to solve complicated priority inversion problems as well as basic one. Our proposed protocol is implemented and tested in the Linux kernel. Additionally, the performance of our proposed protocol is evaluated and compared with previous protocols in the aspect of time and space complexity analyses.
\end{abstract}

\section{Introduction}

Nowadays, real-time operating systems are widely used in various fields where each task must finish execution by its deadline and should meet several requirements such as effective scheduling policies, reducing interrupt handling time, solving priority inversion problems, and so on. Among them, in this paper, the priority inversion problem [1, which means the situation that a high-priority process waits for acquiring the resource that is already locked by a low-priority process, is focused on. This situation occurs sometimes in real-time environments but certainly should be solved to remove the bad effects to the systems. The various solutions to this problem have been presented, among which two recommended protocols are basic priority inheritance (hereafter, BPI) protocol [2] 3] 4] and priority ceiling emulation (hereafter, PCE) protocol [5] 6] 7]. However, they cannot solve some complicated problems, such as the cases when a process locks several resources and when resources are locked and requested recursively. In this paper, a protocol, called recursive priority inheritance (hereafter, RPI) protocol, is proposed. The RPI protocol efficiently solves complicated priority inversion problems as well as basic one, just requiring low time/space complexity compared with pre-existing protocols.

\footnotetext{
* This research was supported by the MIC(Ministry of Information and Communication), Korea, under the ITRC(Information Technology Research Center) support program supervised by the IITA(Institute of Information Technology Advancement) (IITA-2006-C1090-0603-0027).
} 


\section{RPI Protocol}

RPI protocol can solve the complicated priority inversion problems using recursive data structure, which is assigned to each individual process and keeps the entire information on both the resources locked by the process and the processes requesting these resources. (see Figure 1). (Let $P_{x}$ denote processes, and $R_{x}$ a resource; each process has different priority, and the priority of $P_{0}$ is the lowest.)

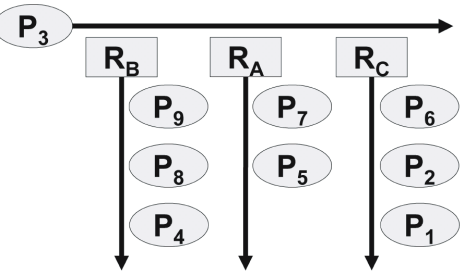

Fig. 1. Conceptual recursive data structure of a process $P_{3}$

As can be seen, $P_{3}$ locks three resources $R_{A}, R_{B}$, and $R_{C}$, and each resource has its own waiting queue for all processes that requested the resource. Processes in the waiting queue of each resource are sorted in the order of their priorities, and the resources locked by $P_{3}$ are also sorted in the order of priorities of the first processes in their waiting queues. Therefore, the first process in the waiting queue of the first resource has always the highest priority among the processes in the queues, and $P_{3}$ inherits the priority of the process; that is, $P_{3}$ inherits the highest priority.

Basically, RPI protocol uses several data structures: a recursive data structure and three new data structures. The former is implemented by adding a few fields to the task_struct structure, which is a structure that contains the entire information on the corresponding process in the Linux kernel. This data structure, a new task_struct structure, is initialized in copy_process() kernel function, which is invoked when a process is created. Additionally, our proposed protocol uses two operations, $P() / V()$, related a semaphore to implement the operations of 'locking' and 'unlocking' a resource. Operating systems, such as Unix and Linux, which support POSIX standards uses semop() system call for $P() / V()$ operations of a semaphore. In the Linux kernel, semop() system call invokes the sys_semtimedop() kernel function, and we modified this function so that it can perform what we want when $P() / V()$ operations are called.

\section{Test and Evaluation}

Our proposed RPI protocol can solve the problem when resources are locked and requested recursively; this problem cannot be solved by previously recommended protocols. (see Figure 2). 


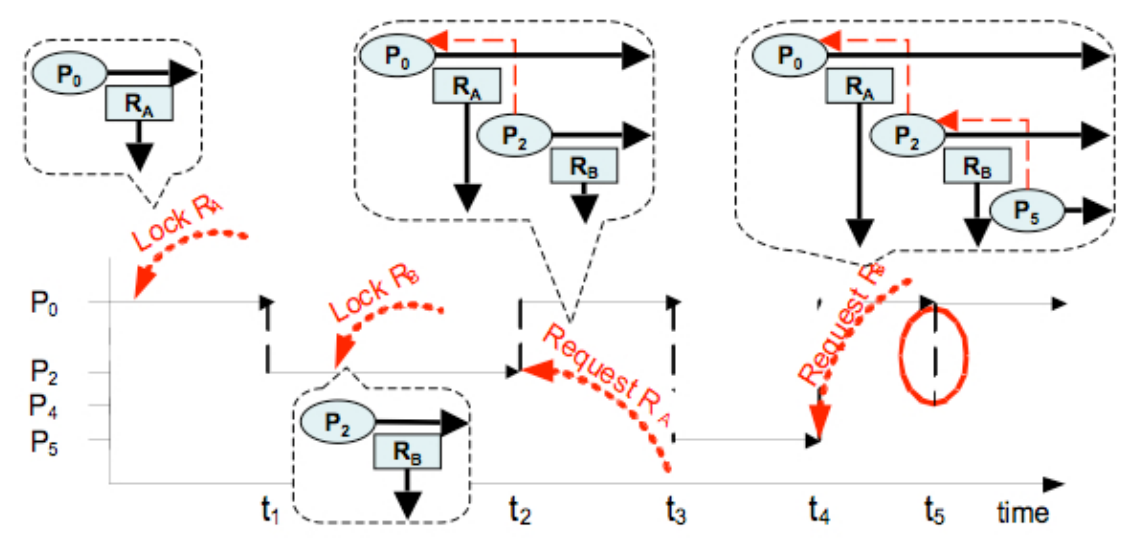

Fig. 2. A procedure when resources are locked and requested recursively

As can be seen, $P_{0}$ and $P_{2}$ lock $R_{A}$ and $R_{B}$, respectively. At time $t_{2}$ when $P_{2}$ requests $R_{A}, P_{0}$ inherits the priority of $P_{2}$, and at time $t_{4}$ when $P_{5}$ requests $R_{B}$, $P_{2}$ inherits the priority of $P_{5}$. In the aspect of $P_{0}$, the priority of $P_{2}$ is changed, and so, that of $P_{0}$ is also changed. In result, at time $t_{5}$ when $P_{4}$ starts to run, $P_{0}$ is not preempted by $P_{4}$ because $P_{0}$ inherits the priority of $P_{5}$.

Now, we test and validate our proposed RPI protocol. The priorities of processes are changed using setpriority() system call which uses internally set_user_nice() kernel function, and $P() / V()$ operations are performed using semget()/semop() system calls, respectively. We assume that the respective priorities of $P_{0}, P_{2}$, and $P_{5}$ are 10, 0 , and -10 in all our tests; the priority of $P_{0}$ is the lowest while that of $P_{5}$ is the highest. In order to customize a general Linux kernel to a real-time operating system, uCLinux patch is applied to the Linux kernel of version 2.6.12 [8]. In this real-time environment, we define a resource as a semaphore and 'lock' (resource request)/'unlock' (resource release) as $P() / V()$ operations of the semaphore, respectively. In addition, although we can directly modify the priorities of the processes in the system, we used the method that modifies the priorities of the processes using set_user_nice() kernel function, which modifies the nice value of a process; the priorities of processes may be modified by other factors, such as scheduling policy.

Comparison criteria are categorized into whether to solve the priority inversion problems or not and the time/space complexity for priority inheritance. ( $n$ and $m$ are the number of processes and resources, respectively) (see Table 1)

As can be seen, our proposed RPI protocol can solve both the basic priority inversion problem and the complicated problems, however, incurs additional expense because the recursive data structure of each process is realigned whenever a process requests or releases a resource. Therefore, the time complexity for priority inheritance is $O(n+m)$. Additionally, each process maintains a recursive data structure for the processes or the resources involved, so the space complexity is also $O(n+m)$. In result, our proposed RPI protocol uses additional data 
Table 1. Comparisons of the RPI protocol with BPI/PCE protocols

\begin{tabular}{c|c|c|c|c}
\hline \multicolumn{2}{l|}{} & BPI protocol & PCE protocol & Proposed protocol \\
\hline \hline \multicolumn{2}{c|}{ Basic problem } & $\mathrm{O}$ & $\mathrm{O}$ & $\mathrm{O}$ \\
\hline \multirow{2}{*}{ Complicated problems } & Case I $^{a}$ & $\mathrm{X}$ & $\mathrm{O}$ & $\mathrm{O}$ \\
\cline { 2 - 5 } & Case II $^{b}$ & $\mathrm{X}$ & $\mathrm{X}$ & $\mathrm{O}$ \\
\hline \multirow{2}{*}{ Complexity } & Time & $O(n)$ & $O(n m)$ & $O(n+m)$ \\
\cline { 2 - 5 } & Space & $O(1)$ & $O(m)$ & $O(n+m)$ \\
\hline
\end{tabular}

a Case I: when a process locks several resources

$b$ Case II: when resources are locked and requested recursively

structure than both the previous two protocols and takes additional time than BPI protocol. However, our protocol can solve complicated priority inversion problems which previous protocols could not have solved as well as basic one.

\section{Conclusion}

Various priority inversion problems are addressed to design and implement realtime operating systems, where each task must finish execution by its deadline. In this paper, RPI protocol was proposed, which solves basic or complicated problems while pre-existing protocols cannot solve the complicated problems completely. The proposed protocol is implemented in the Linux kernel and validated with some test cases. Additionally, the performance of the proposed system is analyzed and compared with the previous recommended protocols.

\section{References}

1. TimeSys Inc., Priority Inversion: Why You Care and What to Do About It. A White Paper, 2004.

2. L. Sha, R. Rajkumar, and J. P. Lehoczky, "Priority Inheritance Protocols: An Approach to Real-Time Synchronization," IEEE Transactions on Computers, Vol. 39 No. 9, Sep. 1990.

3. B. Akgul, V. Mooney, H. Thane, and P. Kuacharoen, "Hardware Support for Priority Inheritance," Proc. of the IEEE Real-Time Systems Symp., Dec. 2003.

4. D. Zöbel, D. Polock, and A. Van Arkel, "Testing for the Conformance of Realtime Protocols Implemented by Operating Systems," Electronic Notes in Theoretical Computer Science, Vol. 133, May 2005.

5. J. B. Goodenough and L. Sha, "The Priority Ceiling Protocol," Special Report CMU/SEI-88-SR-4, Mar. 1998.

6. B. Dutertre, "Formal Analysis of the Priority Ceiling Protocol," Proc. of IEEE Real-Time Systems Symp., Nov. 2000.

7. V. Yodaiken, "Against Priority Inheritance, " FSMLABS Technical Paper, 2003.

8. Embedded Linux/Microcontroller Project, http://www.uclinux.org. 